\title{
Acupuncture in Post-Stroke Shoulder Pain Syndrome with Multiple Sclerosis: A Case Study
}

\author{
Jia Hui Gan ${ }^{1, *}$, Lorenzo A. Santorelli ${ }^{2}$ \\ ${ }^{1}$ Haslemere Hospital, Royal Surrey NHS Foundation Trust, Surrey, United Kingdom \\ ${ }^{2}$ School of Biosciences and Medicine, University of Surrey, Guildford, United Kingdom
}

Received March 28, 2020

Revised September 4, 2020

Accepted December 28, 2020

Correspondence to Jia Hui Gan

Inpatient Therapy, Haslemere Hospital, Haslemere, United Kingdom

E-mail jiahuigan1@gmail.com
The present case study illustrates the case of a 47-year-old female (Ms X) with primary progressive Multiple Sclerosis (MS) who presented with central post-stroke pain (CPSP) over her left shoulder and underwent acupuncture treatment (AT) since she appeared irresponsive to conventional treatment. The aim of this case study is to explore the effectiveness of acupuncture as a complimentary treatment in improving central neurogenic pain in MS patients affected by CPSP. AT lasted six weeks, some modification of the conventional AT points was required to ensure continuity and safety of the treatment plan. In fact, Ms X suffered from gingivitis that led to hypersensitivity of her left upper limb (UL) to acupuncture needling; moreover, she experienced sensation loss in her legs as a result of post-stroke complications. The outcome showed that the subject's shoulder range of motion (ROM), Disabilities of the Arm, Shoulder and Hand (DASH) score and pain improved remarkably, enabling $\mathrm{Ms} \mathrm{X}$ to resume post-stroke rehabilitation and reduce her analgesic intake.

Keywords: Acupuncture Treatment (AT), Central Post-Stroke Pain (CPSP), Shoulder Pain (SP), Multiple Sclerosis (MS)

\section{INTRODUCTION}

Multiple sclerosis is a chronic immune-mediated disease of the central nervous system (CNS) and is one of the most common non-traumatic causes of disability in young adults [1]. Pain is one of the most commonly reported MS symptoms caused by inflammatory demyelination lesions to the white matter, cortex and spinal cord in CNS [2]. Ms X presented CPSP with underlying MS demyelination changes suggesting a central neuropathic pain origin. This caused distressing unrelenting pain that did not allow her to participate in poststroke rehabilitation Upper Limb (UL) therapy. The objective of the present case study is to explore the effectiveness of acupuncture treatment (AT) as a complimentary treatment in improving CPSP with MS background once the mainstream treatments proved ineffective.

\section{CASE PRESENTATION}

The present case study refers to a 47-year-old female (Ms $\mathrm{X}$ ) with background of MS, who presented with severe left shoulder pain for three months following a right middle cerebral artery infarct and underwent an AT programme. The patient did not report any significant past medical condition other than 8 years of primary progressive MS and hypothyroidism. Ms X was working as a part-time administrator and was undergoing out-patient rehabilitation to recover from a stroke. Ms X's medications include an anticoagulant, thyroid hormone replacement therapy, analgesics and antidepressants because of newly diagnosed post-stroke anxiety and depression. Following screening for AT suitability, Ms X underwent once a week acupuncture treatment for six weeks.

\section{Assessment}

On examination, Ms X's left UL muscle atrophy was prominent, displaying hypersensitivity which had hindered manual therapy and post-stroke UL rehabilitation exercise performed by physiotherapist. Oxford muscle strength scale of left shoulder muscles group scored 1/5, elbow and wrists muscles group scored $2 / 5$ respectively with severe movement associated pain. Her left bicep muscle increased tone was 1 based on Modified Ashworth Scale. Ms X scored 87.5 in DASH questionnaire suggested that she experienced severe 
difficulty in most of her daily activities involving of her left UL [3] (further detailed assessment data is available in Appendix 1 and Supplementary Material). Ms X considered the AT as a means to reduce her left UL pain and her dependence on analgesics, enabling her to spend time with her family playing board games and art and craft activities.

A pain Visual Analogue Score was utilised to record the subjective intensity of the pain felt by the subject. The subject was asked to describe the level of pain related to her left shoulder. This was reported using a scale from 0 (no pain) to 10 (extreme pain). The pain intensity assessment was carried out before and after each acupuncture treatment, and it was categorised into two types: resting pain: with no movement required; and movement pain: subject was required to briefly flex her left shoulder.

Ms X central somatosensory pain processing system was believed to be affected as she scored 2/10 in Douleur Neuropathique questionnaire [4] and negative red flag screening. In summary, Ms X presented left UL central neurogenic pain with spasticity despite undergoing three months of intensive rehabilitation programme.

\section{Treatment}

A tailored treatment plan was formulated to address Ms X's complex central somatosensory UL pain disorder. Ms $\mathrm{X}$ had been frustrated since she had unsuccessfully tried several combined treatments including analgesics and transcutaneous electrical nerve stimulator (TENS) in addition to intensive rehabilitation programme. A tailored individualised AT plan was implemented by stimulating various spinal segmental and extra-segmental pathways to reduce segmental and neighbouring segmental hypersensitivity in response to exercise treatment (Appendix 1) [5].

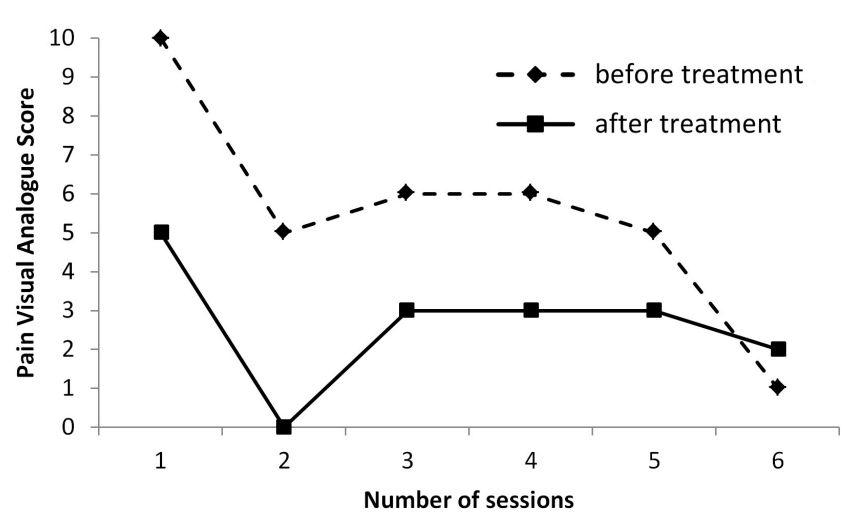

Fig. 1. Change in the subject's left shoulder flexion movement pain intensity before (dotted line) and after (solid line) each acupuncture session. A pain Visual Analogue Score is measured by asking the subject to verbally rate her pain using a scale from 0 (no pain) to 10 (extreme pain). The subject showed a reduction in pain after each session; after six weekly treatments, the pain was recorded as mild $(2 / 10)$.
The authors had initially considered using bilateral LR3 and LI4 points to form "Four Gates" and relieve supraspinal pain; however, safety consideration in "Four Gates" acupuncture in Ms X was limited by her left lower limb poststroke sensation loss. Contralateral acupuncture can prove effective in treating post-stroke shoulder pain, which in turn excites the meridians over the affected left shoulder while considering hypoesthesia effect of stroke [6]. In this case the Zhong ping point was applied to replace left LR3 [7] as it is an influential point to relax shoulder tendons over right leg.

During her fifth treatment, Ms X was unable to tolerate local points on her left UL which could be related to sudden onset of severe gingivitis inflammatory pain. Contralateral acupuncture is commonly used in cases with stroke, however, Kim et al. (2010) [8] suggested that there is limited evidence that contralateral acupuncture is superior to ipsilateral acupuncture. As a result, during her fifth session, instead of applying segmental local points on left UL as usual, the same contralateral points were applied (on the right UL) to target a central effect by activating spinal or supraspinal sympathetic reflexes [9].

\section{Treatment Outcomes}

Ms X's pain levels improved significantly over 6 weeks of treatment enabling her to regain shoulder ROM (Appendix 1, Fig. 1 and 2, Supplementary Material), thus enabling her to undergo post stroke UL rehabilitation from the third week of AT. Such an improvement made it possible to reduce and eventually weaned her off codeine (opiate) intake and allowed her to switch to an occasional intake of paracetamol (Appendix 1). Following six weeks of AT, Ms X was able to enjoy board games as well as art and craft with her family.

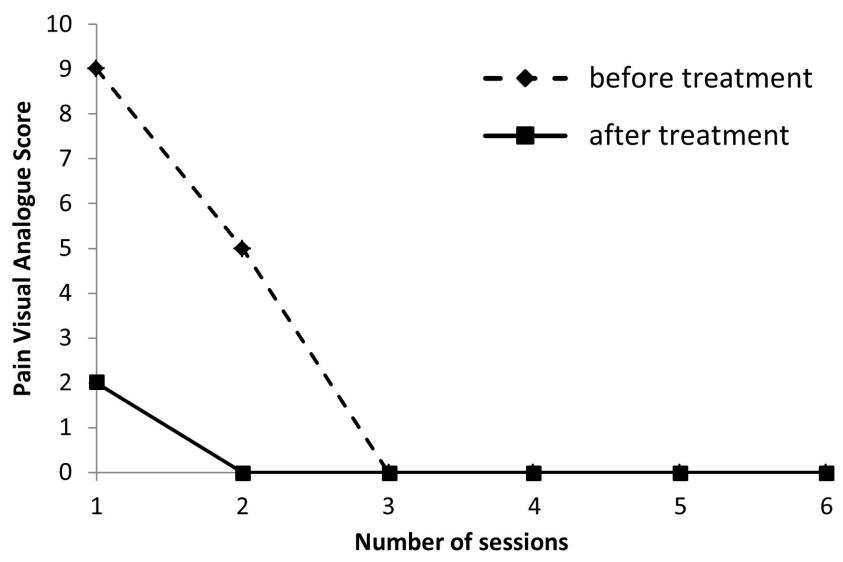

Fig. 2. Change in the subject's left shoulder resting pain intensity before (dotted line) and after (solid line) each acupuncture session. A pain Visual Analogue Score is measured by asking the subject to verbally rate her pain using a scale from 0 (no pain) to 10 (extreme pain). The subject showed a quick reduction in resting pain after the first acupuncture session, with full resolution after the third session. 
The reduced UL pain and function improvement reflected in her DASH score which went from 87.50 to 60.83 (a 30\% drop), exceeding the minimal clinically important difference values of 10.83 points (see Supplementary Material) [3].

\section{DISCUSSION}

Anecdotal evidence on the effectiveness of AT in shoulder pain is available but relevant research in CPSP remains limited [10]. A six week study, combining vigorous acupuncture needling technique and massage therapy in CPSP, suggested that combined therapy can alleviate pain, improve UL functional movement compared to rehabilitation group without acupuncture [11]. Vigorous reinforcingreducing needling technique advocated in this study was attempted on Ms X but was not well tolerated despite of lack of 'De Qi' gained during the first three treatment sessions.

Following four sessions of AT, 'DeQi' was attained with tandem improvement in the bicep tone, which suggests that AT could have improved involuntary pain protective responses in CPSP [12]. A randomized controlled trial on AT in pain-free chronic stroke cases also demonstrated improvement in UL spasticity, but it was limited by a small sample size and multiple, unadjusted, post hoc comparisons [13]. Conversely, a recent systematic review reported that various analgesics and apipuncture had no effect in reducing CPSP associated pain [14].

A different case study proposed a multimodal stepped care approached with both analgesic and acupuncture in treating chronic central origin pain caused by MS [15]. In this case study, Ms X's severe shoulder pain appeared to respond well to added AT to UL rehabilitation with a reduction of analgesic use. LI10 or LI11 points could be applied in this case study to enhance the effectiveness of AT through a mechanotransduction mechanism along the large intestine meridian from UL proximal to distal. However, Ms X was able to tolerate a maximum of 9 acupuncture points per session and the evaluation of the specific effect of particular acupuncture points is beyond the remit of the present case study.

\section{CONCLUSIONS}

The present case study offers an initial insight into the effectiveness of acupuncture as an adjunct treatment in managing severe shoulder pain in CPSP with an MS background. Given the nature of this study on one participant only, it would be inappropriate to infer that this patient would not have recovered without receiving acupuncture if she had continued with conventional rehabilitation. For a better generalisability of the results reported in this case study, future randomized controlled trials comparing acupuncture with rehabilitation to conventional rehabilitation group are needed. A lack of evidence in similar rare case, it is crucial to cite the principles of acupuncture treatment and its therapeutic outcomes in balancing pain while undergoing intensive post-stroke rehabilitation management.

\section{SUPPLEMENTARY MATERIAL}

Supplementary data to this article can be found online at https://doi.org/10.51507/j.jams.2021.14.1.27

\section{ACKNOWLEDGEMENTS}

I would like to thank to Ms X and her family in their commitment to complete six weeks of acupuncture treatment without extra incentive.

\section{CONFLICT OF INTEREST}

The authors declare no conflict of interest.

\section{ORCID}

Jia Hui Gan, https://orcid.org/0000-0003-4122-3166 Lorenzo A. Santorelli,

https://orcid.org/0000-0002-8392-801X

\section{REFERENCES}

1. Dutta R, Trapp BD. Relapsing and progressive forms of multiple sclerosis: insights from pathology. Curr Opin Neurol 2014;27: 271-8.

2. Khan N, Smith MT. Multiple sclerosis-induced neuropathic pain: pharmacological management and pathophysiological insights from rodent EAE models. Inflammopharmacology 2014;22:1-22.

3. Franchignoni F, Vercelli S, Giordano A, Sartorio F, Bravini E, Ferriero G. Minimal clinically important difference of the disabilities of the arm, shoulder and hand outcome measure (DASH) and its shortened version (QuickDASH). J Orthop Sports Phys Ther 2014;44:30-9.

4. Bouhassira D, Attal N, Alchaar H, Boureau F, Brochet B, Bruxelle J, et al. Comparison of pain syndromes associated with nervous or somatic lesions and development of a new neuropathic pain diagnostic questionnaire (DN4). Pain 2005; 114:29-36.

5. Zhao ZQ. Neural mechanism underlying acupuncture analgesia. Prog Neurobiol 2008;85:355-75.

6. Sun KO, Chan KC, Lo SL, Fong DY. Acupuncture for frozen shoulder. Hong Kong Med J 2001;7:381-91.

7. Wang W, Yin X, He Y, Wei J, Wang J, Di F. Treatment of 
periarthritis of the shoulder with acupuncture at the zhongping (foot) extrapoint in 345 cases. J Tradit Chin Med 1990;10:20912.

8. Kim MK, Choi TY, Lee MS, Lee H, Han CH. Contralateral acupuncture versus ipsilateral acupuncture in the rehabilitation of post-stroke hemiplegic patients: a systematic review. BMC Complement Altern Med 2010;10:41.

9. Bradnam L. A proposed clinical reasoning model for Western acupuncture. NZJP 2003;31:40-5.

10. Lee JA, Park SW, Hwang PW, Lim SM, Kook S, Choi KI, et al. Acupuncture for shoulder pain after stroke: a systematic review. J Altern Complement Med 2012;18:818-23.

11. Li N, Tian F, Wang C, Yu P, Zhou X, Wen Q, et al. Therapeutic effect of acupuncture and massage for shoulder-hand syndrome in hemiplegia patients: a clinical two-center randomized controlled trial. J Tradit Chin Med 2012;32:343-9.

12. Vickers A, Zollman C. ABC of complementary medicine. Acupuncture. BMJ 1999;319:973-6.

13. Wayne PM, Krebs DE, Macklin EA, Schnyer R, Kaptchuk TJ, Parker SW, et al. Acupuncture for upper-extremity rehabilitation in chronic stroke: a randomized sham-controlled study. Arch Phys Med Rehabil 2005;86:2248-55.

14. Mulla SM, Wang L, Khokhar R, Izhar Z, Agarwal A, Couban R, et al. Management of central poststroke pain: systematic review of randomized controlled trials. Stroke 2015;46:2853-60.

15. Kopsky DJ, Hesselink JM. Multimodal stepped care approach with acupuncture and PPAR- $\alpha$ agonist palmitoylethanolamide in the treatment of a patient with multiple sclerosis and central neuropathic pain. Acupunct Med 2012;30:53-5. 